\title{
Conjugated Polymers as a New Class of Dual-Mode Matrices for MALDI Mass Spectrometry and Imaging
}

\author{
Kilian Horatz, ${ }^{\dagger, \ddagger}$ Marco Giampà, ${ }^{\S}$ Yevhen Karpov, ${ }^{\dagger}$ Karin Sahre, ${ }^{\dagger}$ Hanna Bednarz, ${ }^{\S}$ Anton Kiriy, ${ }^{\dagger}$ \\ Brigitte Voit, ${ }^{\dagger}$ Karsten Niehaus, ${ }^{\S}$ Nikos Hadjichristidis, ${ }^{\circledR}$ Dominik L. Michels, ${ }^{\perp}$ \\ and Franziska Lissel* ${ }^{*} \dagger$ (0)
}

†Institute of Macromolecular Chemistry, Leibniz-Institut für Polymerforschung Dresden e.V., Hohe Straße 6, 01069 Dresden, Germany

${ }^{\ddagger}$ Organic Chemistry of Polymers, Technische Universität Dresden, Mommsenstrasse 4, 01062 Dresden, Germany

${ }^{\S}$ Center for Biotechnology and Department for Proteome and Metabolome Research, Faculty of Biology, Bielefeld University, Universitätsstraße 25, 33615 Bielefeld, Germany

\#Physical Sciences and Engineering Division, KAUST Catalysis Center, Polymer Synthesis Laboratory, King Abdullah University of Science and Technology (KAUST), Thuwal 23955, Kingdom of Saudi Arabia

${ }^{\perp}$ Computer, Electrical and Mathematical Sciences and Engineering Division, KAUST Visual Computing Center, Computational Sciences Group, King Abdullah University of Science and Technology (KAUST), Thuwal 23955, Kingdom of Saudi Arabia

\section{Supporting Information}

\begin{abstract}
Matrix-assisted laser desorption/ionization mass spectrometry (MALDI MS) and MALDI MS imaging are ubiquitous analytical methods in medical, pharmaceutical, biological, and environmental research. Currently, there is a strong interest in the investigation of low molecular weight compounds (LMWCs), especially to trace and understand metabolic pathways, requiring the development of new matrix systems that have favorable optical properties and a high ionization efficiency and that are MALDI silent in the LMWC area. In this paper, five conjugated polymers, poly $\left\{\left[N, N^{\prime}-\right.\right.$ bis(2-octyldodecyl)-naphtalene-1,4,5,8-bis(dicarboximide)2,6-diyl]-alt-5,5'(2,2'-bithiophene)\} (PNDI(T2)), poly(3-do-

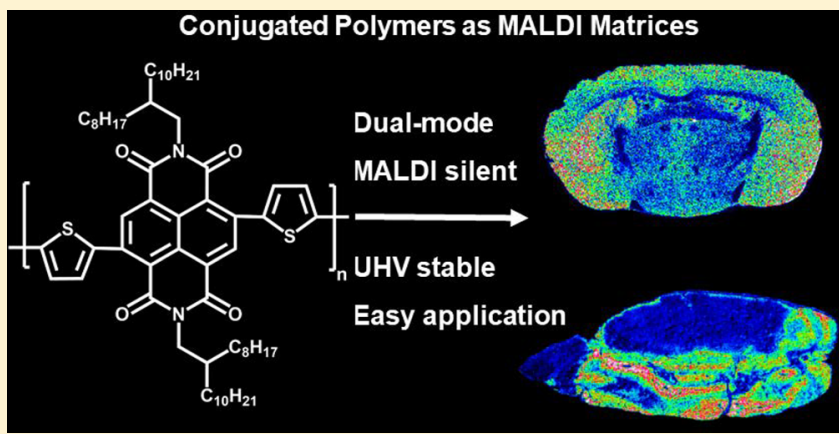
decylthiophene-2,5-diyl) (P3DDT), poly\{[2,3-bis(3-octyloxyphenyl)quinoxaline-5,8-diyl]-alt-(thiophene-2,5-diyl)\} (PTQ1), poly $\left\{\left[N, N^{\prime}\right.\right.$-bis(2-octyldodecyl)-isoindigo-5,5'-diyl]-alt-5,5'(2,2'-bithiophene) $\}$ (PII(T2)), and poly(9,9-di- $n$-octylfluorenyl-2,7diyl) (P9OFl) are investigated as matrices. The polymers have a strong optical absorption, are solution processable, and can be coated into thin films, allowing a vast reduction in the amount of matrix used. All investigated polymers function as matrices in both positive and negative mode MALDI, classifying them as rare dual-mode matrices, and show a very good analyte ionization ability in both modes. PNDI(T2), P3DDT, PTQ1, and PII(T2) are MALDI silent in the full measurement range $(>m / z=$ $150 \mathrm{k})$, except at high laser intensities. In MALDI MS experiments of single analytes and a complex biological sample, the performance of the polymers was found to be as good as two commonly used matrices (2,5-DHB for positive and 9AA for negative mode measurements). The detection limit of two standard analytes was determined as being below 164 pmol for reserpine and below 245 pmol for cholic acid. Additionally P3DDT was used successfully in first MALDI MS imaging experiments allowing the visualization of the tissue morphology of rat brain sections.
\end{abstract}

\section{INTRODUCTION}

MALDI MS and MALDI MS Imaging. Since the development of "matrix-assisted laser desorption/ionization mass spectrometry" (MALDI MS) in the $1980 \mathrm{~s},{ }^{1-3}$ this type of mass spectrometry evolved into an important analysis tool for medical and biochemical investigations. ${ }^{4-8}$ Compared with other types of MS, e.g., electrospray ionization (ESI), secondary ion mass spectrometry (SIMS), or fast atom bombardment (FAB), MALDI MS exhibits several advantages: As the analyte is integrated into a protecting matrix, it is softly ionized, and high molecular weight compounds (e.g., proteins, lipids, synthetic polymers) can be measured without destroying or fragmentizing them. ${ }^{9,10}$ The method does not require the labeling of the analytic targets and permits measurements down to attomolar concentrations.

MALDI mass spectrometry imaging (MS imaging) is an emerging clinicopathological imaging tool based on MALDI MS. $^{1{ }^{1-13}}$ It enables determining and visualizing the spatial distribution of molecules in a tissue sample, for example, in a

Received: June 23, 2018

Published: August 8, 2018 
Scheme 1. Molecular Structures of (a) Poly\{[N,N'-bis(2-octyldodecyl)-naphthalene-1,4,5,8-bis(dicarboximide)-2,6-diyl]-alt5,5'-(2,2'-bithiophene)\} (PNDI(T2)), (b) Poly(3-dodecylthiophene-2,5-diyl) (P3DDT), (c) Poly\{[2,3-bis(3octyloxyphenyl)quinoxaline-5,8-diyl]-alt-(thiophene-2,5-diyl) $\}$ (PTQ1), (d) Poly $\left\{\left[N, N^{\prime}\right.\right.$-bis(2-octyldodecyl)-isoindigo-5,5'diyl]-alt-5,5'-(2,2'-bithiophene)\} (PII(T2)), and (e) Poly(9,9-di-n-octylfluorenyl-2,7-diyl) (P9OFl)

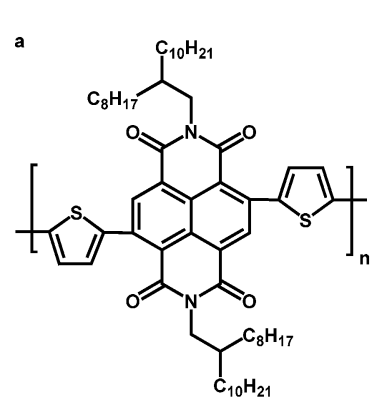

PNDI(T2)

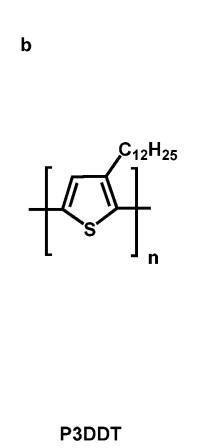

P3DDT

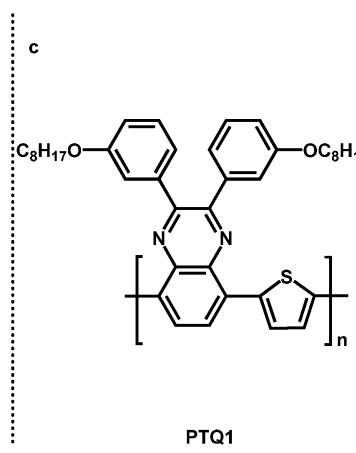

PTQ1

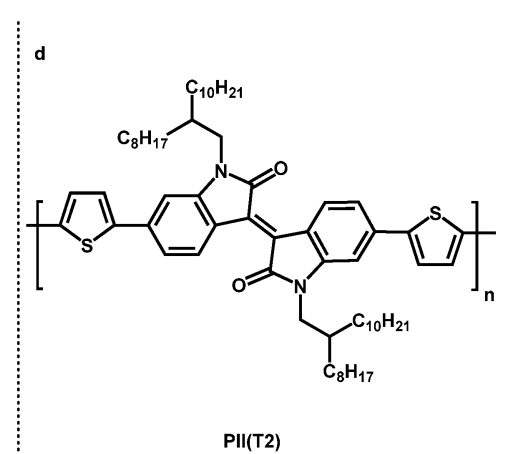

PII(T2)

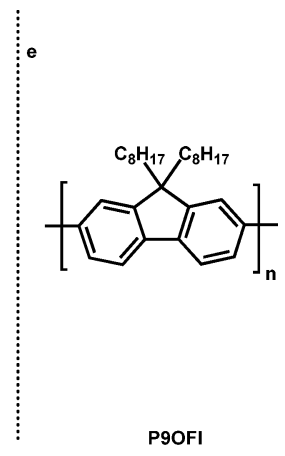

P9OFI cancer section, and is a versatile method with a high potential for medical, ${ }^{14,15}$ pharmaceutical, ${ }^{16}$ and environmental ${ }^{17}$ research. MALDI MS imaging and MALDI MS share the same working principles, but where MALDI MS establishes which molecular masses are present in the sample as a whole, MALDI MS imaging introduces spatial resolution by coating the matrix on the surface of a sample and then taking a series of measurements along a predefined grid. Each collected spectrum gives a detailed summary of the molecules present at the grid point, and combining the spectra allows the visualization of concentration gradients of molecules of interest and the creation of molecular maps of the measured tissue. It is also possible to construct $3 \mathrm{D}$ molecular maps by dividing an organ into several slices and merging all recorded spectra. ${ }^{18}$

Currently there is a drive to establish MALDI protocols for the analysis of low molecular weight compounds (LMWCs). ${ }^{17}$ This interest can be attributed to intensified efforts to study the metabolic profile of biological and pathological samples. The bulk of metabolites have a low molecular weight, and tracing their occurrence and composition is an important angle in the research on the formation and progression of diseases such as cancer, ${ }^{16}$ in drug development, ${ }^{15}$ or for the detection ${ }^{17}$ of pollutants and toxins.

Matrix Requirements. Choosing the right matrix for a given sample or analyte is a crucial step in the MALDI process. The ideal matrix has a strong optical absorption in the spectral region of the commonly used UV and IR lasers and a high ionization efficiency and is "MALDI silent", i.e., does not lead to matrix-related peaks in the spectrum. ${ }^{12,19}$

A MALDI MS imaging matrix ideally meets two additional conditions. $^{12,20}$ (1) Homogenous matrix layer: The commonly used crystalline matrices need to exhibit a crystal density sufficient to allow measurements of the complete surface without gap regions, and the individual crystal has to be small relative to the grid size to avoid creating images that contain artifacts, as the lateral resolution is limited by the size of the matrix crystals. (2) Increased vacuum stability: Due to the high number of collected spectra, MALDI MS imaging measurements can take several hours to complete, especially in highmass and/or high lateral resolution modes. To ensure comparability between different samples and also different positions on the same sample, the matrix cover not only needs to be highly uniform but also needs to be fully stable in ultrahigh vacuum (UHV) over an extended period of time.
Current state-of-the-art matrices were developed for specific analytes in regular MALDI MS using a "trial-and-error" approach. ${ }^{12,19}$ They are usually small molecules with conjugated systems and acidic or basic functional groups, e.g., 2,5-dihydroxybenzoic acid (2,5-DHB), ${ }^{21}$ 3,5-dimethoxy-4hydroxycinnamic acid (sinapinic acid), $\alpha$-cyano-4-hydroxycinnamic acid ( $\alpha$-CHCA), and so on. These small-molecule matrices desorb and ionize themselves, leading to matrixrelated signals in the low molecular weight part of the MS spectrum. Due to the commonly used high matrix/analyte ratio, the matrix-related peaks can be intense and the resulting spectral interference as well as detector saturation often impedes the analysis of LMWCs. ${ }^{19}$ Several methods were explored to avoid the interferences from the matrix in the lowmass range. One is to change the ratio of analyte and matrix in order to have a complete suppression of matrix peaks; ${ }^{22,23}$ another way is to derivatize selected targets to shift their mass to a higher mass range where matrix-related peaks are not present. $^{24-26}$

Most well-established matrices are suitable for either positive or negative mode measurements, but not for both. For samples available only in low quantity, e.g., fine needle biopsies, or for studies investigating numerous samples in high-throughput analyses, it would be furthermore advantageous to have matrix systems available that are "dual mode" and allow high-quality measurements in both positive and negative mode. ${ }^{27}$ As yet, only a few matrices are known that enable such dual-mode measurements. ${ }^{28-30}$ Furthermore, some of the small molecular matrices exhibit a comparably low vacuum stability, which influences the homogeneity of the layer especially during longterm MALDI MS imaging measurements and can falsify the resulting ion maps.

Consequently the development of new matrix systems is necessary to solve these problems. ${ }^{19,31,32}$ Rational design and synthesis of new crystalline small-molecule matrices as well as the deployment of novel compound classes, e.g., graphene, ${ }^{33,34}$ graphene oxide, $^{34,35}$ nanoparticles, ${ }^{36}$ metal oxides, ${ }^{37}$ or ionic liquids, ${ }^{38}$ are currently explored.

Conjugated Polymers as Matrices. MALDI MS is a standard tool for the analysis of polymeric materials. ${ }^{39-41}$ The utilization of polymers as MALDI matrices instead of small molecules could solve the described matrix problems: Conjugated polymers have a large $\pi$-system enabling them to interact with light of different wavelengths, which allows a versatile utilization of the matrices with different instruments 
Table 1. UV/Vis Absorption of the Investigated Polymers

\begin{tabular}{|c|c|c|c|c|c|}
\hline & PNDI(T2) & P3DDT & PTQ1 & PII(T2) & P9OFl \\
\hline $\begin{array}{l}\text { absorption maxima } \\
{[\mathrm{nm}]^{a, b}}\end{array}$ & $\begin{array}{l}392(100 \%), 697 \\
(81 \%)\end{array}$ & $\begin{array}{l}523(100 \%), 549(95 \%), 601 \\
(61 \%)\end{array}$ & $\begin{array}{l}363(95 \%), 622 \\
(100 \%)\end{array}$ & $\begin{array}{l}413(67 \%), 626(100 \%), 686 \\
(89 \%)\end{array}$ & $\begin{array}{l}386(100 \%), 432 \\
(13 \%)\end{array}$ \\
\hline $\begin{array}{l}\text { relative absorption at } 355 \\
n^{b}\end{array}$ & $82 \%$ & $23 \%$ & $93 \%$ & $48 \%$ & $72 \%$ \\
\hline
\end{tabular}

and laser wavelengths. Due to their high molecular weight and chemical stability, they are nonvolatile and potentially allow measurement of spectra without matrix peaks (MALDI silent). Standard conjugated polymers used in organic electronics carry alkyl side chains, are soluble in organic solvents, and can be coated into semicrystalline thin films with a surface roughness in the low nanometer range using established methods, ${ }^{42,43}$ enabling homogeneous and defined matrix layers without gap regions. The molecular structure of the polymer backbone influences the physical properties (e.g., optical absorption, vacuum stability), and the polymer side chains can be used to modulate the chemical properties (e.g., solubility, analyte extraction ability). The large variability of the backbone and the possibility to introduce functional side chains, together with their overall ease of processing, make conjugated polymers very promising candidates to realize a novel class of functional high-performance matrices for MALDI MS and MALDI MS imaging. Interestingly, the research into polymers as functional matrix systems is so far limited to one report using poly(3-octylthiophene-2,5-diyl) for the measurements of acids. ${ }^{44}$ Investigations of other polymer systems have not been reported, and the potential of polymers as functional dualmode matrices as well as the measurement of complex samples was never explored. Also, to the best of our knowledge, polymer matrices have never been used in MALDI MS imaging.

In this paper, five different conjugated polymers are investigated regarding their suitability as matrices for both negative and positive MALDI measurements. The polymers (see Scheme 1) poly\{[N,N'-bis(2-octyldodecyl)-naphthalene1,4,5,8-bis(dicarboximide)-2,5-diyl]-alt-5,5'-(2,2'bithiophene)\} (PNDI(T2)), poly(3-dodecylthiophene-2,5-diyl) (P3DDT), poly\{[2,3-bis(3-octyloxyphenyl)quinoxaline-5,8-diyl]-alt-(thiophene-2,5-diyl) $\}$ (PTQ1), poly $\left\{\left[N, N^{\prime}\right.\right.$-bis(2-octyldodecyl)-isoindigo-5,5'-diyl]-alt-5,5'-(2,2'-bithiophene)\} (PII(T2)), and poly(9,9-di- $n$-octylfluorenyl-2,7-diyl) (P9OFl) are well-explored semiconducting polymers. UV/vis spectra of dropcasted polymer films are recorded to determine the absorption at the MALDI laser wavelength, and the polymers are measured as analytes in MALDI experiments with and without secondary matrix to investigate their behavior. Then the performance of the polymers as matrices is tested in MALDI measurements of different pure LMWC analytes as well as of a complex biological sample and compared against two wellestablished matrices, 2,5-DHB for positive mode and 9AA for negative mode MALDI MS. The detection limit of standard analytes in both positive and negative mode is determined. Finally, P3DDT is tested in MALDI MS imaging experiments.

\section{EXPERIMENTAL SECTION}

MALDI MS spectra were recorded with a Bruker Autoflex speed MALDI-TOF/TOF equipped with a Smartbeam-II Nd:YAG laser $(355 \mathrm{~nm})$. The software tools flexControl 3.3 and flexAnalysis 3.3 were used. All MS measurements of analytes were carried out in reflectron mode, and the instrument was calibrated with red phosphorus $^{45}$ at the beginning of each measurement cycle (see Supporting Information for further details). PNDI(T2 $)^{42}$ and PII(T2) ${ }^{46}$ were synthesized in our laboratory; all other compounds are commercially available. All polymers except PTQ1 were purified with Soxhlet extraction before use. Layers of analytes and matrices were drop-coated from suitable solvents using the thin layer method, a variation of the dried droplet method (see Supporting Information). ${ }^{47,48}$ The polymers were coated from toluene solutions. For each matrix the best layer thickness combination was qualitatively determined by testing several combinations of analyte and matrix concentration (see Supporting Information for further details). The MALDI MS imaging experiments were carried out in negative reflectron mode on a Bruker ultrafleXtreme MALDI-TOF instrument equipped with a Smartbeam-II Nd:YAG laser (355 nm) and using flexImaging 4.1 software. P3DDT was spray-coated from an acetonitrile/chloroform solution using a Bruker ImagePrep instrument. All experimental details are described in the Supporting Information.

\section{RESULTS AND DISCUSSION}

Favorable optical properties are one of the basic prerequisites for MALDI matrices, and small-molecule matrices are often chosen based on an absorption maximum close to the laser wavelength (e.g., 2,5-DHB: local maximum at $324 \mathrm{~nm}$ with $18 \%$ of $\left.\lambda_{\max }=208 \mathrm{~nm}\right)^{21,49}$ All investigated polymers show good to very high relative absorptions $A_{\mathrm{Nd} \text { :YAG }}$ at $355 \mathrm{~nm}$, the wavelength of the commonly used Nd:YAG laser (Table 1 and Figure S4), thereby meeting the optical criteria. PNDI(T2) $\left(\lambda_{\max }=392 \mathrm{~nm}, A_{\mathrm{Nd}: \mathrm{YAG}}=82 \%\right)$, PTQ1 $\left(\lambda_{\max }=622 \mathrm{~nm}\right.$, $\left.A_{\mathrm{Nd}: \mathrm{YAG}}=93 \%\right)$, and P9OFl $\left(\lambda_{\max }=386 \mathrm{~nm}, A_{\mathrm{Nd}: \mathrm{YAG}}=72 \%\right)$ have very good optical properties with regard to the wavelength of the Nd:YAG laser, while PII(T2) $\left(\lambda_{\max }=626\right.$ $\left.\mathrm{nm}, A_{\mathrm{Nd}: \mathrm{YAG}}=48 \%\right)$ and P3DDT $\left(\lambda_{\max }=523 \mathrm{~nm}, A_{\mathrm{Nd}: \mathrm{YAG}}=\right.$ $23 \%)$ absorb less strongly, but still sufficiently well.

MALDI experiments can be carried out in linear or reflectron mode. The former allows measuring up to high molecular weights, but enables only a limited resolution and is commonly used for the analysis of polymers, while the reflectron mode permits a higher resolution but a limited $\mathrm{m} / z$ range and is preferred for metabolomics and peptidomics analyses and for MALDI MS imaging experiments.

To analyze the integrity of polymer structure, MALDI measurements of the polymers as analytes were carried out in positive linear mode using trans-2-[3-(4-tert-butylphenyl)-2methyl-2-propenylidene]malononitrile (DCTB) as secondary matrix under the addition of 1 vol \% of sodium trifluoroacetate (NaTFA), and the resulting spectra show typical patterns in the range up to $m / z=14 \mathrm{k}$ (Figures S78-S87) associated with the molecular mass distributions. Please note that in the corresponding figures the range below $m / z=1000$ is deliberately not shown, as the intense DCTB-related peaks would dwarf the peaks of the polymeric analytes (Figure S77). The linear mode measurements were carried out with a laser intensity of $80 \%$, which is significantly higher than the intensities used for the later polymer matrix experiments. 
Positive and negative MALDI experiments of only the polymers without secondary matrix were carried out in linear as well as reflectron mode. Without a secondary matrix, PNDI(T2), P3DDT, PTQ1, and PII(T2) are MALDI silent in both positive and negative linear mode unless very high laser intensities are used. Similar results are obtained in positive and negative reflectron mode measurements: At low laser intensities the matrices are MALDI silent in both positive and negative mode (Figures S88 and S90), and only for high laser intensities are alkyl chain fragments visible at $m / z \leq 85$ (Figures S89 and S91).

In positive reflectron mode, alkali cations were visible at $\mathrm{m} / \mathrm{z}$ $=23\left(\mathrm{Na}^{+}\right)$and $39\left(\mathrm{~K}^{+}\right)$in some measurements, while in negative reflectron mode halide ions were sometimes detected at $m / z=39\left(\mathrm{Cl}^{-}\right)$and $79 / 81\left(\mathrm{Br}^{-}\right)$. These ions were observed already for measurements of the pure sample plate and are commonly found artifacts. While 2,5-DHB and 9AA tend to form clusters with these ions, thereby leading to more peaks in the LMWC range (Figures S5-S10), this was not observed for the polymer matrices.

Even without secondary matrix, P9OFl shows polymerrelated patterns in both positive and negative mode measurements (Figures S88-S91). Test measurements with pure analytes using $\mathrm{P} 9 \mathrm{OFl}$ as matrix in both positive and negative reflectron mode allowed the detection of the analytes, but also showed peaks relating to the polymer backbone (Figures S35 and S36). The polymer was not further investigated.

All measurements using the polymers as matrix were carried out in the high-resolution reflectron mode commonly used for metabolic analyses and MALDI MS imaging experiments. Analytes and polymer matrices were deposited in layers on the sample plate using the thin layer method ${ }^{47,48}$ with the matrix coated on the analyte. This sample preparation corresponds to the device structure commonly found in MALDI MS imaging experiments, ${ }^{12}$ and coating the matrix as a thin film on the analyte requires significantly less matrix material compared to the classic sample preparation by cocrystallizing (mixing). Comparing layered and mixed sample preparation revealed that the sensitivity of the former outperforms the latter (Figures S92-S95).

Positive Reflectron Mode. Three compounds were measured in positive reflectron mode MALDI MS to assess the potential of the polymeric matrices, and the results of these experiments are compared against 2,5-DHB: reserpine (RP), an indole alkaloid used as MS standard, tetraethylammonium chloride (TEAC), which is used in histological assays to block potassium channels, and coumaphos (CP), a phosphorothioate-based insecticide used to control varroa mite infestation in honeybee colonies (see Table S2 for the analyte structures).

The results of these measurements are visualized in graphs containing four stacked spectra depicting, top down, (a) only the sample plate, (b) a layer of pure analyte without matrix, (c) a layer of pure matrix without analyte, and (d) an analyte layer coated with a matrix layer (see Figure 1). All polymers allow MALDI MS measurements of the analytes in positive and negative mode (see Figures 1 and 2 and Supporting Information). Comparing spectra (b) and (d) shows that the polymer is essential for successful measurements, as without it the analytes are not detected.

PNDI(T2), P3DDT, and PTQ1 were drop-coated from solutions with a very low concentration of $0.1 \mathrm{mg} / \mathrm{mL}$, while for PII(T2) a concentration of $10 \mathrm{mg} / \mathrm{mL}$ was used (see

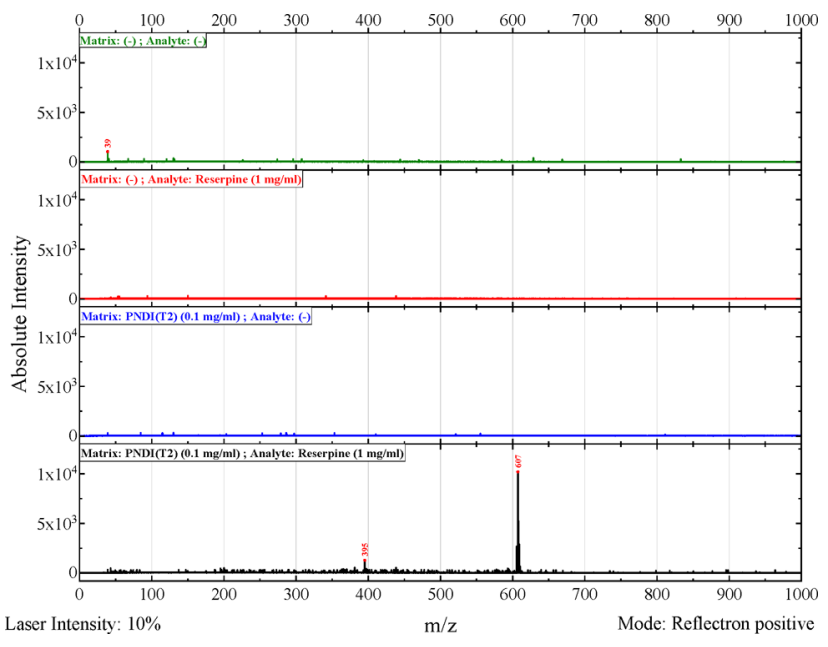

Figure 1. Reflectron positive mode MALDI MS spectra of (a) sample plate (green), (b) only the pure analyte RP (red), (c) only the pure matrix PNDI(T2) (blue), and (d) PNDI(T2) coated on RP (black). Peaks: $m / z=607\left[\mathrm{RP}-\mathrm{H}^{-}\right]^{+}$; $395 \mathrm{RP}$ fragment $[\mathrm{RP} 3]^{+}$; $39[\mathrm{~K}]^{+}$.

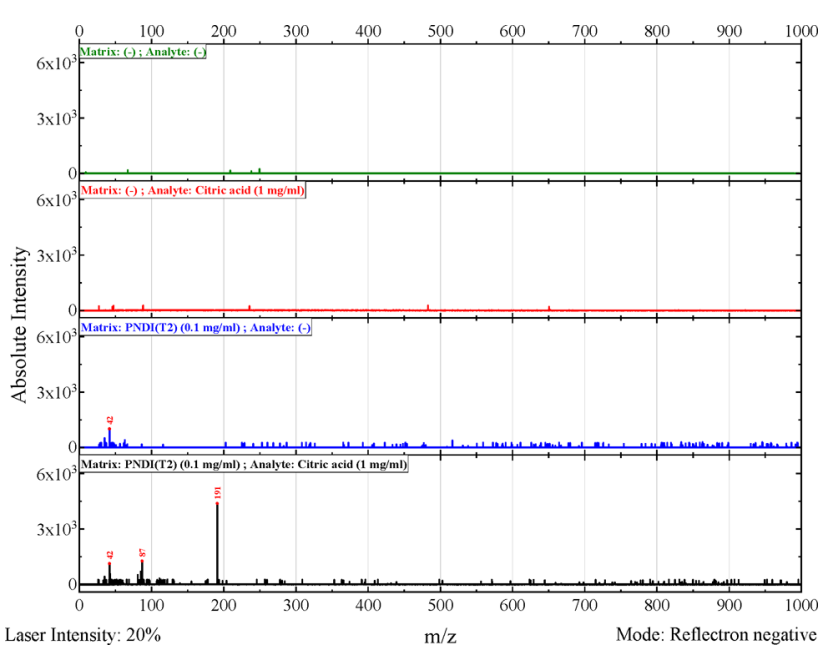

Figure 2. Reflectron negative mode MALDI MS spectra of (a) sample plate (green), (b) only the pure analyte CiA (red), (c) only the pure matrix PNDI(T2) (blue), and (d) PNDI(T2) coated on CiA (black). Peaks: $m / z=191\left[\mathrm{CiA}-\mathrm{H}^{+}\right]^{-} ; 87 \mathrm{CiA}$ fragment $[\mathrm{CiAl}]^{-}$; 42 side chain fragment $\left[\mathrm{C}_{3} \mathrm{H}_{6}\right]^{-}$.

Supporting Information for further information). Reserpine was measured with low laser intensities between $10 \%$ and $40 \%$ (PNDI(T2) Figure S11; P3DDT Figure S17; PTQ1 Figure S23; PII(T2) Figure S29). The already reported phenomenon of hydride elimination was observed in all polymer measurements, leading to a molecular mass signal at $\mathrm{m} / z=607 .^{50}$ In addition to this peak, RP fragments are observable in the PNDI(T2) and P3DDT measurements.

TEAC was detected as $[\mathrm{TEA}]^{+}$at very low laser intensities of 10-20\% (PNDI(T2) Figure S12; P3DDT Figure S18; PTQ1 Figure S24; PII(T2) Figure S30). Interestingly, in both PNDI(T2) and P3DDT measurements a fragment is observable despite a laser intensity of $10 \%$, while both PTQ1 and PII(T2) experiments with $20 \%$ solely show the molecular mass peak of [TEA $]^{+}$at $m / z=130$.

In comparison to RP and TEAC, the detection of $\mathrm{CP}$ required higher laser intensities of $20-50 \%$ for all four polymer matrices (PNDI(T2) Figure S13; P3DDT Figure S19; PTQ1 
Figure S25; PII(T2) Figure S31). Fragments of CP were not detectable, but in the PTQ1 measurement, a peak at $\mathrm{m} / z=$ 401 could correspond to $\left[\mathrm{M}+\mathrm{K}^{+}\right]^{+}$.

Negative Reflectron Mode. For the assessment of the polymer matrices in negative reflectron mode MALDI MS, three metabolites were investigated (see Table S2 for molecular structures): cholic acid (ChA), an important primary bile acid with a steroid structure; citric acid ( $\mathrm{CiA}$ ), the central intermediate of the citric acid cycle; and Lphenylalanine (PA), an essential amino acid monitored in newborns to detect phenylketonuria. The results of the polymer matrix measurements were compared against measurements using 9AA.

The experiments establish that the polymers are essential for the detection of the analytes under the investigated conditions and substantiate that PNDI(T2), P3DDT, PTQ1, and PII(T2) function as negative mode matrices, classifying them as new dual-mode matrices. P9OFl similarly allows analyte detection in both modes, but was not further investigated due to the observed polymer-related peaks.

PII(T2), PNDI(T2), and PTQ1 were coated from solutions with a very low concentration of $0.1 \mathrm{mg} / \mathrm{mL}$, and P3DDT was coated with a concentration of $1 \mathrm{mg} / \mathrm{mL}$ (see Supporting Information for further information).

ChA was measured with laser intensities of $30-40 \%$, and the molecular mass peak was detected in every experiment. Also alkyl side chains at $m / z=42$ and 57 are visible (PNDI(T2) Figure S14; P3DDT Figure S20; PTQ1 Figure S26; PII(T2) Figure S32).

$\mathrm{CiA}$ was detected with very low laser intensities of $10-30 \%$ (PNDI(T2) Figure 2; P3DDT Figure S21; PTQ1 Figure S27; PII(T2) Figure S33). A fragment of CiA is observable in the spectra of PNDI(T2), PTQ1, and PII(T2) at $m / z=87$.

The measurements of PA were carried out with laser intensities of $30-40 \%$. PTQ1 gives a very good spectrum (Figure S28) containing solely the molecular mass peak. In the spectra of PII(T2), PNDI(T2), and P3DDT additionally lowintensity peaks relating to alkyl side chains are visible at $\mathrm{m} / z=$ 42 and 57 (PNDI(T2) Figure S16; P3DDT Figure S22; PII(T2) Figure S34).

It has to be noted that optical properties and MALDI matrix suitability (required laser intensities, analyte ionization ability) are not directly correlated. While in both positive and negative mode there is a clear trend that higher laser intensities cause more distinct side chain signals, the intensity threshold seems to be influenced by several factors.

Detection Limit. Using the polymer matrices PNDI(T2), P3DDT, PTQ1, and PII(T2), concentration-dependent studies were carried out in both positive and negative reflectron mode MALDI MS to evaluate the sensitivity of the polymer matrices and the potential for quantitative analyses. Similarly to the previous measurements, first the analyte was drop-coated from a solution of a defined concentration; then the polymer was coated over the analyte. The concentration of the analyte was stepwise reduced until a detection was no longer possible, while all experimental parameters were kept constant. In positive mode, RP was measured with a laser intensity of $20 \%$ (PNDI(T2)), $40 \%$ (P3DDT) and 30\% (PTQ1, PII(T2)). The limit of detection (LOD) for RP was found to be below 16 pmol for PNDI(T2) and below 164 pmol for P3DDT, PTQ1, and PII(T2) under the chosen conditions (PNDI(T2): Figures S53-S55; P3DDT: Figures S59-S61; PTQ1: Figures S65-S67; PII-
(T2): Figures S71-S73). Using a laser intensity of $50 \%$ (PNDI(T2), PII(T2)) and 30\% (P3DDT, PTQ1), ChA was similarly measured in the negative mode, and the LOD was found to be below 245 pmol for all polymer matrices (PNDI(T2): Figures S56-58; P3DDT: Figures S62-S64; PTQ1: Figures S68-S70; PII(T2): Figures S74-S76). Both LOD values were confirmed in three independent measurement series. Although the measurement conditions were not optimized, all polymer matrices show a very good sensitivity in both positive and negative mode LOD experiments, further substantiating the potential of conjugated polymers as excellent dual-mode matrices.

Complex Sample. To determine whether the polymer matrices are also suitable to measure complex biological samples containing a high number of metabolites in low individual concentration, a commercially available Gingko biloba (G.B.) extract was investigated in both positive and negative mode reflectron MALDI MS with PNDI(T2), P3DDT, PTQ1, and PII(T2). G.B. was chosen as complex sample as the extract contains a rich variety of flavonoids and terpene lactones, and studies ${ }^{51-53}$ using different MS methods identified several key components. All four investigated polymers allow recording complex spectra of G.B. in both positive and negative mode (PNDI(T2) Figures 3 and 4;

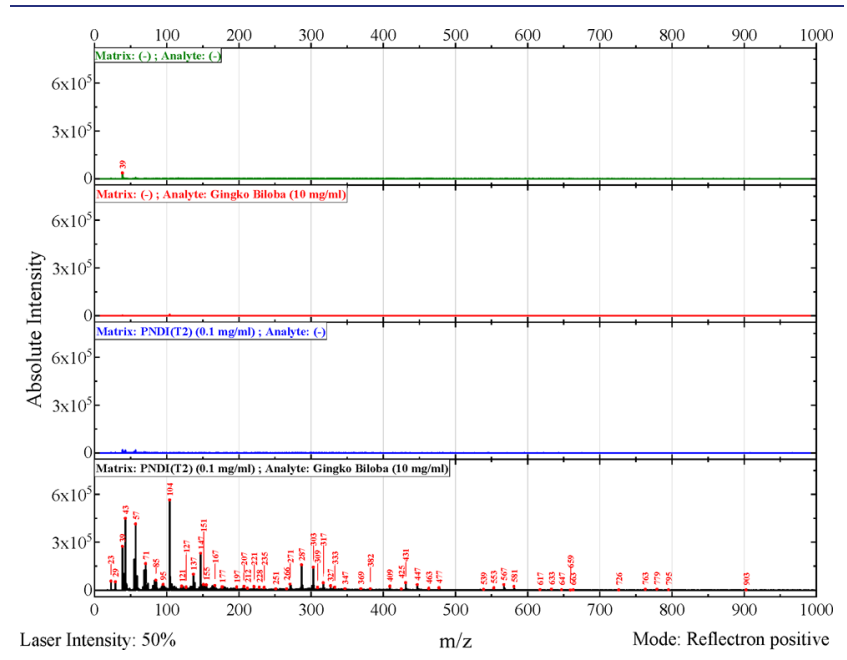

Figure 3. Reflectron positive mode MALDI MS spectra of (a) sample plate (green), (b) only the analyte G.B. (red), (c) only the pure matrix PNDI(T2) (blue), and (d) PNDI(T2) coated on G.B. (black). $m / z=39$ corresponds to $[\mathrm{K}]^{+}$.

P3DDT Figures S44-S46; PTQ1 Figures S47-S49; PII(T2) Figure S50-S52), confirming the suitability of the conjugated polymers for the dual-mode analysis of biological samples. The peak count and peak intensity were further analyzed.

In the positive mode, the number of detected peaks (with $I$ $>100$ ) and the average peak intensity using either of the polymers are fully consistent with the results obtained with 2,5DHB (see Table 2, Figures S37 and S38). When the intensity threshold is raised $(I>10 \mathrm{k})$, the number of detected peaks is in the same range for 2,5-DHB and PNDI(T2) (120 and 125, respectively), while P3DDT, PTQ1, and PII(T2) have significantly fewer high-intensity peaks $(26,20$, and 43, respectively) and consequently achieve a better average peak intensity.

The prepared samples were subsequently used for negative mode measurements. With regard to the number of detected 


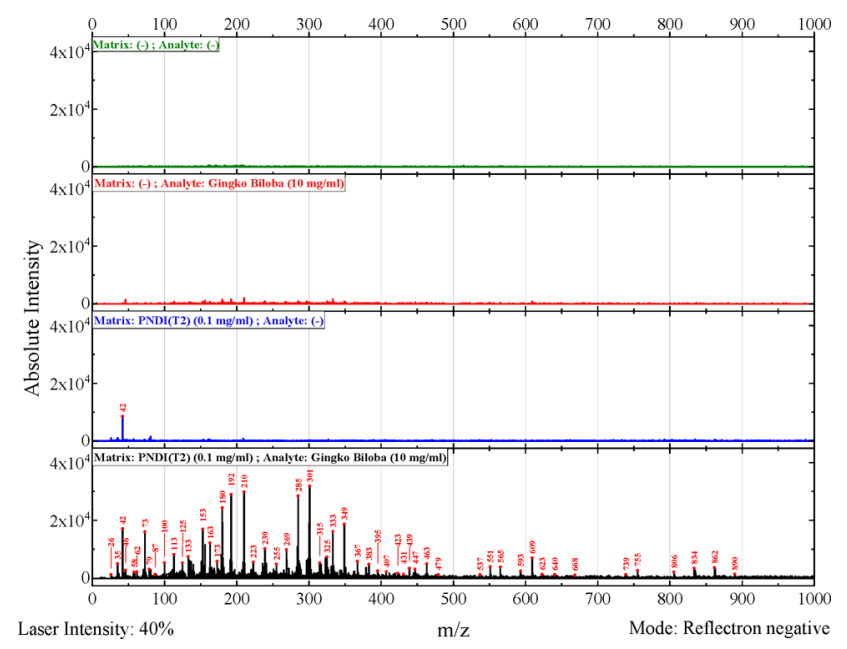

Figure 4. Reflectron negative mode MALDI MS spectra of (a) sample plate (green), (b) only the analyte G.B. (red), (c) only the pure matrix PNDI(T2) (blue), and (d) PNDI(T2) coated on G.B. (black). Peaks: $m / z=79 / 81[\mathrm{Br}]^{-} ; 42$ side chain fragment $\left[\mathrm{C}_{3} \mathrm{H}_{6}\right]^{-}$.

peaks and the average peak intensity, PII(T2), PNDI(T2), and PTQ1 are comparable to 9AA (see Table 2, Figures S39 and S40), while P3DDT records about $25 \%$ less peaks, but significantly more high-intensity peaks (50\% increase). Again PII(T2), PNDI(T2), and PTQ1 lead to fewer high-intensity peaks but an improved average peak intensity.

The experiments substantiate that the investigated polymers are efficient dual-mode matrices and suitable for the analysis of complex biological samples. It has to be noted that in the measurements with 2,5-DHB and 9AA the most intense peaks are caused by the small-molecule matrices.

MALDI MS Imaging. First MALDI MS imaging experiments of fresh-frozen rat brain sections ${ }^{54}$ using P3DDT as matrix were performed. One coronal and one sagittal section were spray-coated with the polymer and measured in reflectron negative mode. Each section was sampled at approximately 85834 and 51445 individual locations or pixels for sagittal and coronal, respectively. Each pixel corresponds to a full mass spectrum with an $m / z$ range of $0-2000$.

Figure 5 depicts heat maps visualizing the spatial distribution of eight different ions in a single coronal brain section. The ions have masses ranging from 101.0 $\mathrm{Da}( \pm 0.3 \mathrm{Da})$ to 904.7 $\mathrm{Da}( \pm 0.3 \mathrm{Da})$, thereby covering the range of LMWCs central to metabolomics. In Figure 6, the spatial distributions of eight different ions in a single sagittal brain section are visualized. In order to represent the broad range of detected metabolites, the depicted ions have molecular masses from 95.9 Da $( \pm 0.1 \mathrm{Da})$ to $904.5 \mathrm{Da}( \pm 0.1 \mathrm{Da})$.

In both data sets, different brain regions are clearly distinguishable, and the visualization results are well correlated to the tissue morphology, confirming the high potential of the used conjugated polymers as matrices in MALDI MS imaging. Indeed, the combination of the high molecular weight and the resulting stability together with the extensive $\pi$-conjugation and high UV absorption of the polymer leads to a complete absence of matrix-related peaks in the $\mathrm{m} / \mathrm{z}$ range of $60-2000$ in the mass spectra. This causes a significant improvement of the detection and evaluation of tissue-specific ions in the data set.

\section{CONCLUSIONS AND OUTLOOK}

MALDI MS is a ubiquitous analytical tool, and MALDI MS imaging has a high potential to become a standard imaging method in medical, pharmaceutical, and biological research. Both methods are increasingly used for the analysis of LMWCs, for example, metabolites, requiring new matrix systems that do not lead to matrix-related signals in the lowmolecular-weight region and have an increased vacuum stability.

In this paper, five different conjugated polymers, PNDI(T2), P3DDT, PTQ1, PII(T2), and P9OFl, were tested as matrix systems in positive and negative mode MALDI MS. All investigated polymers have a high molecular weight and are fully vacuum stable. They are solution processable, can be coated into thin films, and show good to very good absorption behavior in the optical range of the commonly used Nd:YAG laser, thereby meeting all prerequisites for a MALDI matrix.

Using defined LMWCs as well as a complex Gingko biloba extract as analytes, we could show that all polymers function as matrices for positive as well as negative mode MALDI, classifying them as rare dual-mode matrices. Drop-coating thin films of the polymers on the analyte in a variation of the thin layer method allowed a vast reduction in the amount of matrix used without compromising the analytical result.

PNDI(T2), P3DDT, PTQ1, and PII(T2) are MALDI silent in the full measurement range in both positive (upper instrument limit $m / z=200 \mathrm{k}$ ) and negative mode (upper instrument limit $m / z=160 \mathrm{k}$ ), except at high laser intensities, when peaks at $m / z \leq 85$ are visible, which are attributable to fragments (in source decay) of the solubilizing alkyl chains. P9OFl allows the detection of analytes, but as the main chain of the polymer fragmentizes and backbone-related peaks are visible in both positive and negative mode spectra, this polymer was not further investigated. The performance of

Table 2. Number of Peaks and Average Peak Intensity in Reflectron Mode Measurements of G.B.

\begin{tabular}{|c|c|c|c|c|c|c|}
\hline \multirow[b]{2}{*}{ matrix } & \multicolumn{3}{|c|}{ reflectron positive mode } & \multicolumn{3}{|c|}{ reflectron negative mode } \\
\hline & $\begin{array}{c}\text { no. of peaks } P_{100} \text { with I } \\
>100 \text { (av peak } \\
\text { intensity) }\end{array}$ & $\begin{array}{c}\text { no. of peaks } P_{10 \mathrm{k}} \text { with } I \\
>10 \mathrm{k} \text { (av peak } \\
\text { intensity) }\end{array}$ & $\begin{array}{c}\text { most intense peak } \\
m / z \text { (peak } \\
\text { intensity) }\end{array}$ & $\begin{array}{c}\text { no. of peaks } P_{100} \text { with } I \\
>100 \text { (av peak } \\
\text { intensity) }\end{array}$ & $\begin{array}{c}\text { no. of peaks } P_{10 \mathrm{k}} \text { with } I \\
>\text { 10k (av peak } \\
\text { intensity) }\end{array}$ & $\begin{array}{c}\text { most intense peak } \\
m / z \text { (peak } \\
\text { intensity) }\end{array}$ \\
\hline $\mathrm{DHB}$ & $2980\left(3.5 \times 10^{3}\right)$ & $120\left(4.3 \times 10^{4}\right)$ & $215\left(3.2 \times 10^{5}\right)^{a}$ & & & \\
\hline $9 A A$ & & & & $2972\left(2.7 \times 10^{3}\right)$ & $110\left(3.4 \times 10^{4}\right)$ & $193\left(7.3 \times 10^{5}\right)^{a}$ \\
\hline PNDI(T2) & $2980\left(3.3 \times 10^{3}\right)$ & $125\left(4.4 \times 10^{4}\right)$ & $104\left(5.7 \times 10^{5}\right)$ & $2966\left(7.6 \times 10^{2}\right)$ & $16\left(2.0 \times 10^{4}\right)$ & $301\left(3.2 \times 10^{4}\right)$ \\
\hline P3DDT & $2986\left(2.8 \times 10^{3}\right)$ & $26\left(4.8 \times 10^{4}\right)$ & $104\left(5.0 \times 10^{5}\right)$ & $2260\left(3.3 \times 10^{3}\right)$ & $155\left(3.6 \times 10^{4}\right)$ & $210\left(3.3 \times 10^{5}\right)$ \\
\hline $\operatorname{PII}(\mathrm{T} 2)$ & $2976\left(2.6 \times 10^{3}\right)$ & $43\left(5.4 \times 10^{4}\right)$ & $104\left(6.6 \times 10^{5}\right)$ & $2960\left(7.6 \times 10^{2}\right)$ & $19\left(1.9 \times 10^{4}\right)$ & $210\left(3.5 \times 10^{4}\right)$ \\
\hline PTQ1 & $2977\left(1.8 \times 10^{3}\right)$ & $20\left(5.7 \times 10^{4}\right)$ & $104\left(5.5 \times 10^{5}\right)$ & $2513\left(1.5 \times 10^{3}\right)$ & $66\left(2.8 \times 10^{4}\right)$ & $210\left(1.1 \times 10^{5}\right)$ \\
\hline
\end{tabular}

${ }^{a}$ Matrix-related peak. 


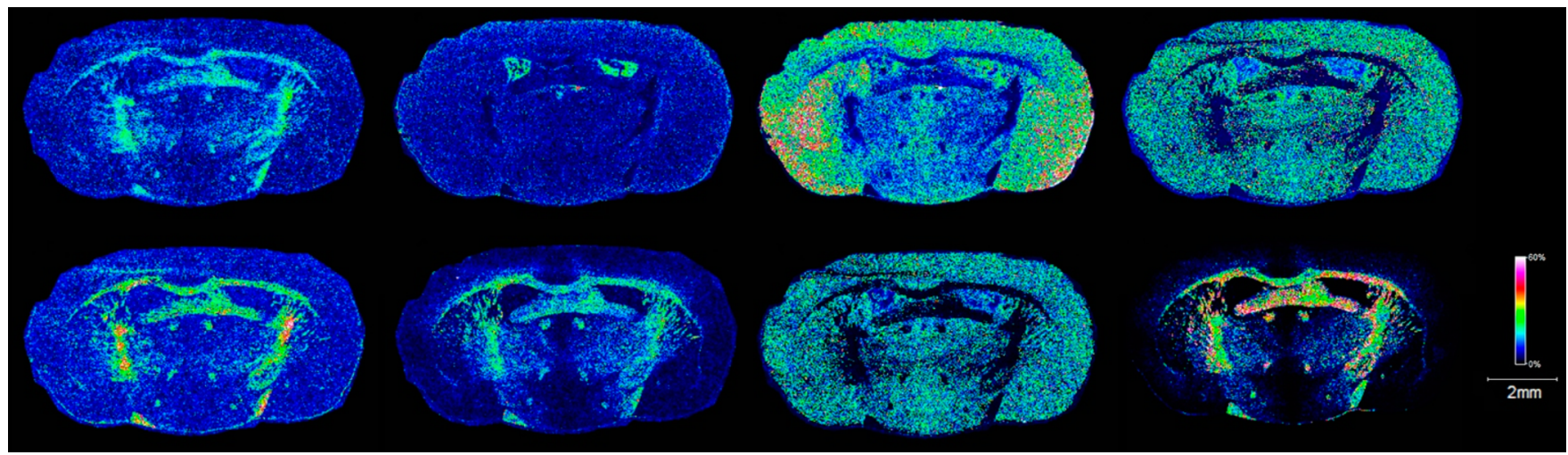

Figure 5. Exemplary ion images of selected mass channels acquired by MALDI MS imaging of a coronal rat brain section in negative mode and by using P3DDT as matrix. The distributions of the ionic species are visualized as heat maps. Ionic species $(\mathrm{m} / \mathrm{z})$ from top left to bottom right: 101.0 $\mathrm{Da}( \pm 0.3 \mathrm{Da}) ; 113.1 \mathrm{Da}( \pm 0.3 \mathrm{Da}) ; 124.0 \mathrm{Da}( \pm 0.2 \mathrm{Da}) ; 210.9 \mathrm{Da}( \pm 0.3 \mathrm{Da}) ; 225.2 \mathrm{Da}( \pm 0.3 \mathrm{Da}) ; 309.5 \mathrm{Da}( \pm 0.3 \mathrm{Da}) ; 384.7 \mathrm{Da}( \pm 0.3 \mathrm{Da})$; $904.7 \mathrm{Da}( \pm 0.3 \mathrm{Da})$.

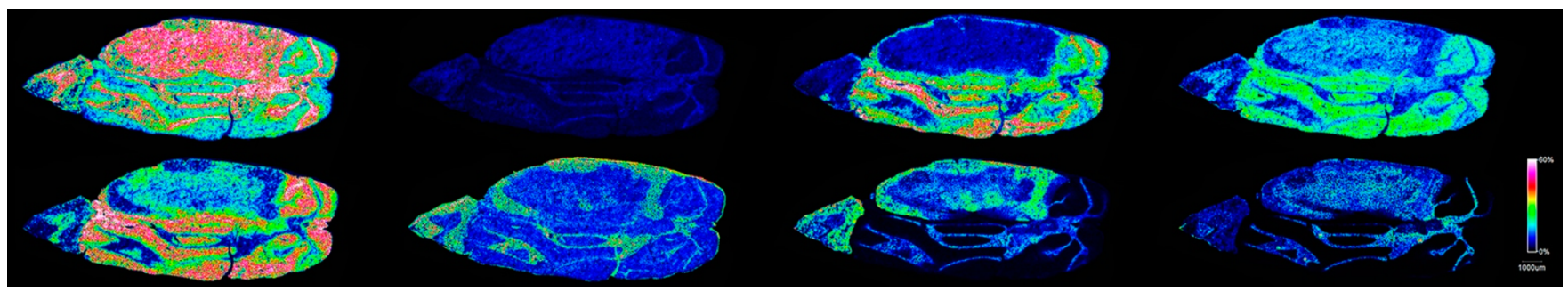

Figure 6. Exemplary ion images of selected mass channels acquired by MALDI MS imaging of a sagittal rat brain section in negative mode and by using P3DDT as matrix. The distributions of the ionic species are visualized as heat maps. Ionic species $(\mathrm{m} / \mathrm{z})$ from top left to bottom right: 97.1 $\mathrm{Da}( \pm 0.3 \mathrm{Da}) ; 113.1 \mathrm{Da}( \pm 0.3 \mathrm{Da}) ; 124.0 \mathrm{Da}( \pm 0.3 \mathrm{Da}) ; 153.1 \mathrm{Da}( \pm 0.3 \mathrm{Da}) ; 181.0 \mathrm{Da}( \pm 0.3 \mathrm{Da}) ; 225.3 \mathrm{Da}( \pm 0.3 \mathrm{Da}) ; 309.3 \mathrm{Da}( \pm 0.1 \mathrm{Da})$; $904.5 \mathrm{Da}( \pm 0.3 \mathrm{Da})$.

PNDI(T2), P3DDT, PTQ1, and PII(T2) as matrices was tested in reflectron mode, which permits a higher resolution and is preferred for metabolomics and MALDI MS imaging experiments. The efficacy when measuring single LMWC analytes is very good and in all cases comparable with or better than the performance of the well-established matrices 2,5-DHB (positive mode) and 9AA (negative mode). The measurement of a G.B. extract as an example of a complex biological sample similarly confirmed that the analyte ionization ability and sensitivity of the polymer matrices are comparable with or better than those of the commonly used matrices 2,5-DHB and 9AA. MALDI MS imaging experiments were performed on coronal and sagittal rat brain sections, and the visualization results are well correlated to the tissue morphology.

In summary, the presented study shows that conjugated polymers are rare dual-mode MALDI matrices combining vacuum stability and a very good analyte ionization ability with being MALDI silent. Due to the inherent structural versatility of both polymer backbone and side chains, conjugated polymers are a highly promising molecule class to design new functional dual-mode matrix systems for both MALDI MS and MALDI MS imaging.

Currently, we are carrying out more detailed studies on the suitability of selected polymer systems as MALDI MS imaging matrices, as well as exploring structural variations to further improve the analyte ionization ability.

\section{ASSOCIATED CONTENT}

\section{S Supporting Information}

The Supporting Information is available free of charge on the ACS Publications website at DOI: 10.1021/jacs.8b06637.

Details about the used chemicals, the employed instrumentation, and experimental settings, as well as results of the UV/vis as well as all MALDI MS experiments (PDF)

\section{AUTHOR INFORMATION}

\section{Corresponding Author}

*lissel@ipfdd.de

\section{ORCID}

Anton Kiriy: 0000-0002-4263-9377

Nikos Hadjichristidis: 0000-0003-1442-1714

Franziska Lissel: 0000-0003-0254-4565

\section{Notes}

The authors declare no competing financial interest.

\section{ACKNOWLEDGMENTS}

The work was supported by KAUST Center Partnership Fund OSR-2017-CPF-3322-01. F.L. thanks the VCI for a Liebig Fellowship. The authors gratefully acknowledge the support of Wolfgang Heidrich in establishing the collaboration infrastructure between KAUST's VCC and the IPF. Anastasia Khrenova is thanked for helping with the graphics. 


\section{REFERENCES}

(1) Karas, M.; Bachmann, D.; Hillenkamp, F. Anal. Chem. 1985, 57, 2935-2939.

(2) Karas, M.; Bachmann, D.; Bahr, U.; Hillenkamp, F. Int. J. Mass Spectrom. Ion Processes 1987, 78, 53-68.

(3) Tanaka, K.; Waki, H.; Ido, Y.; Akita, S.; Yoshida, Y.; Yoshida, T.; Matsuo, T. Rapid Commun. Mass Spectrom. 1988, 2, 151-153.

(4) Clark, A. E.; Kaleta, E. J.; Arora, A.; Wolk, D. M. Clin Microbiol Rev. 2013, 26, 547-603.

(5) Croxatto, A.; Prod'hom, G.; Greub, G. FEMS Microbiol Rev. 2012, 36, 380-407.

(6) Rodrigo, M. A. M.; Zitka, O.; Krizkova, S.; Moulick, A.; Adam, V.; Kizek, R. J. Pharm. Biomed. Anal. 2014, 95, 245-255.

(7) Patel, R. Clin. Chem. 2015, 61, 100-111.

(8) Duncan, M. W.; Nedelkov, D.; Walsh, R.; Hattan, S. J. Clin. Chem. 2016, 62, 134-143.

(9) Karas, M.; Hillenkamp, F. Anal. Chem. 1988, 60, 2299-2301.

(10) Meier, M. A. R.; Hoogenboom, R.; Fijten, M. W. M.; Schneider, M.; Schubert, U. S. J. Comb. Chem. 2003, 5, 369-374.

(11) Chaurand, P.; Stoeckli, M.; Caprioli, R. M. Anal. Chem. 1999, $71,5263-5270$

(12) Norris, J. L.; Caprioli, R. M. Chem. Rev. 2013, 113, 2309-2342.

(13) Crecelius, A. C.; Schubert, U. S.; von Eggeling, F. Analyst 2015, $140,5806-5820$.

(14) Aichler, M.; Walch, A. Lab. Invest. 2015, 95, 422-431.

(15) Rauser, S.; Marquardt, C.; Balluff, B.; Deininger, S.-O.; Albers, C.; Belau, E.; Hartmer, R.; Suckau, D.; Specht, K.; Ebert, M. P.; Schmitt, M.; Aubele, M.; Höfler, H.; Walch, A. J. Proteome Res. 2010, 9, 1854-1863.

(16) Reyzer, M. L.; Hsieh, Y.; Ng, K.; Korfmacher, W. A.; Caprioli, R. M. J. Mass Spectrom. 2003, 38, 1081-1092.

(17) Lagarrigue, M.; Caprioli, R. M.; Pineau, C. J. Proteomics 2016, 144, 133-139.

(18) Seeley, E. H.; Caprioli, R. M. Anal. Chem. 2012, 84, 21052110

(19) Calvano, C. D.; Monopoli, A.; Cataldi, T. R. I.; Palmisano, F. Anal. Bioanal. Chem. 2018, 410, 4015-4038.

(20) Li, S.; Zhang, Y.; Liu, J. A.; Han, J.; Guan, M.; Yang, H.; Lin, Y.; Xiong, S.; Zhao, Z. Sci. Rep. 2016, 6, 37903.

(21) Strupat, K.; Karas, M.; Hillenkamp, F. Int. J. Mass Spectrom. Ion Processes 1991, 111, 89-102.

(22) Knochenmuss, R.; Dubois, F.; Dale, M. J.; Zenobi, R. Rapid Commun. Mass Spectrom. 1996, 10, 871-877.

(23) McCombie, G.; Knochenmuss, R. Anal. Chem. 2004, 76, 49904997.

(24) Monopoli, A.; Calvano, C. D.; Nacci, A.; Palmisano, F. Chem. Commun. (Cambridge, U. K.) 2014, 50, 4322-4324.

(25) Shariatgorji, M.; Nilsson, A.; Källback, P.; Karlsson, O.; Zhang, X.; Svenningsson, P.; Andren, P. E. J. Am. Soc. Mass Spectrom. 2015, 26, 934-939.

(26) Manier, M. L.; Spraggins, J. M.; Reyzer, M. L.; Norris, J. L.; Caprioli, R. M. J. Mass Spectrom. 2014, 49, 665-673.

(27) Janfelt, C.; Wellner, N.; Hansen, H. S.; Hansen, S. H. J. Mass Spectrom. 2013, 48, 361-366.

(28) Anderson, D. M. G.; Ablonczy, Z.; Koutalos, Y.; Spraggins, J.; Crouch, R. K.; Caprioli, R. M.; Schey, K. L. J. Am. Soc. Mass Spectrom. 2014, 25, 1394-1403.

(29) Kettling, H.; Vens-Cappell, S.; Soltwisch, J.; Pirkl, A.; Haier, J.; Müthing, J.; Dreisewerd, K. Anal. Chem. 2014, 86, 7798-7805.

(30) Thomas, A.; Charbonneau, J. L.; Fournaise, E.; Chaurand, P. Anal. Chem. 2012, 84, 2048-2054.

(31) Giampà, M.; Lissel, M. B.; Patschkowski, T.; Fuchser, J.; Hans, V. H.; Gembruch, O.; Bednarz, H.; Niehaus, K. Chem. Commun. (Cambridge, U. K.) 2016, 52, 9801-9804.

(32) Muthu, M.; Chun, S.; Wu, H.-F.; Duncan, M. W.; Gopal, J. J. Mass Spectrom. 2018, 53, 525-540.

(33) Dong, X.; Cheng, J.; Li, J.; Wang, Y. Anal. Chem. 2010, 82, $6208-6214$
(34) Liu, Y.; Liu, J.; Yin, P.; Gao, M.; Deng, C.; Zhang, X. J. Mass Spectrom. 2011, 46, 804-815.

(35) Wang, Z.; Cai, Y.; Wang, Y.; Zhou, X.; Zhang, Y.; Lu, H. Sci. Rep. 2017, 7, 44466.

(36) Marsico, A. L. M.; Creran, B.; Duncan, B.; Elci, S. G.; Jiang, Y.; Onasch, T. B.; Wormhoudt, J.; Rotello, V. M.; Vachet, R. W. J. Am. Soc. Mass Spectrom. 2015, 26, 1931-1937.

(37) Bernier, M. C.; Wysocki, V. H.; Dagan, S. J. Mass Spectrom. 2015, 50, 891-898.

(38) Towers, M. W.; Cramer, R. Ionic Liquids and Other Liquid Matrices for Sensitive MALDI MS Analysis. In Advances in MALDI and Laser-Induced Soft Ionization Mass Spectrometry; Cramer, R., Ed.; Springer International Publishing: Cham, 2016; pp 51-64.

(39) Pasch, H.; Schrepp, W. MALDI-TOF Mass Spectrometry of Synthetic Polymers; Springer Science \& Business Media, 2013.

(40) Oh, J.; Kuk, J.; Lee, T.; Ye, J.; Paik, H.-J.; Lee, H. W.; Chang, T. ACS Macro Lett. 2017, 6, 758-761.

(41) Liu, J.; Loewe, R. S.; McCullough, R. D. Macromolecules 1999, $32,5777-5785$

(42) Tkachov, R.; Karpov, Y.; Senkovskyy, V.; Raguzin, I.; Zessin, J.; Lederer, A.; Stamm, M.; Voit, B.; Beryozkina, T.; Bakulev, V.; Zhao, W.; Facchetti, A.; Kiriy, A. Macromolecules 2014, 47, 3845-3851.

(43) Karpov, Y.; Zhao, W.; Raguzin, I.; Beryozkina, T.; Bakulev, V.; Al-Hussein, M.; Häußler, L.; Stamm, M.; Voit, B.; Facchetti, A.; Tkachov, R.; Kiriy, A. ACS Appl. Mater. Interfaces 2015, 7, 1247812487.

(44) Soltzberg, L. J.; Patel, P. Rapid Commun. Mass Spectrom. 2004, $18,1455-1458$.

(45) Sládková, K.; Houska, J.; Havel, J. Rapid Commun. Mass Spectrom. 2009, 23, 3114-3118.

(46) Karpov, Y.; Maiti, J.; Tkachov, R.; Beryozkina, T.; Bakulev, V.; Liu, W.; Komber, H.; Lappan, U.; Al-Hussein, M.; Stamm, M.; Voit, B.; Kiriy, A. Polym. Chem. 2016, 7, 2691-2697.

(47) Kussmann, M.; Nordhoff, E.; Rahbek-Nielsen, H.; Haebel, S.; Rossel-Larsen, M.; Jakobsen, L.; Gobom, J.; Mirgorodskaya, E.; KrollKristensen, A.; Palml, L.; Roepstorff, P. J. Mass Spectrom. 1997, 32, 593-601.

(48) Ole, V.; Roepstorff, P.; Mann, M. Anal. Chem. 1994, 66, 32813287

(49) Nielsen, K. Journal of Chromatography A 2003, 1002, 111-136. (50) Awad, H.; Stoudemayer, M. J.; Usher, L.; Amster, I. J.; Cohen, A.; Das, U.; Whittal, R. M.; Dimmock, J.; El-Aneed, A. J. Mass Spectrom. 2014, 49, 1139-1147.

(51) Beck, S.; Stengel, J. Phytochemistry 2016, 130, 201-206.

(52) Ding, S.; Dudley, E.; Plummer, S.; Tang, J.; Newton, R. P.; Brenton, A. G. Phytochemistry 2008, 69, 1555-1564.

(53) Ding, S.; Dudley, E.; Song, Q.; Plummer, S.; Tang, J.; Newton, R. P.; Brenton, A. G. Rapid Commun. Mass Spectrom. 2008, 22, 766772.

(54) Animal tissue used in this study was kindly provided by the animal facility of the Bielefeld University. It represents tissues from animals that have been sacrificed in the context of other studies with no connection to the experiments presented in this article. 